Rapport - Société canadienne d'histoire de l'Église catholique

\title{
Paroisses françaises principales de la péninsule de Niagara
}

\section{Augustin Buisson}

Volume 29, 1962

URI : https://id.erudit.org/iderudit/1007361ar

DOI : https://doi.org/10.7202/1007361ar

Aller au sommaire du numéro

Éditeur(s)

La Société canadienne d'histoire de l'Église catholique

ISSN

0318-6148 (imprimé)

1927-7075 (numérique)

Découvrir la revue

Citer cet article

Buisson, A. (1962). Paroisses françaises principales de la péninsule de Niagara. Rapport - Société canadienne d'histoire de l'Église catholique, 29, 11-19.

https://doi.org/10.7202/1007361ar

Tous droits réservés @ La Société canadienne d'histoire de l'Église catholique, 1963
Ce document est protégé par la loi sur le droit d'auteur. L'utilisation des services d'Érudit (y compris la reproduction) est assujettie à sa politique d'utilisation que vous pouvez consulter en ligne.

https://apropos.erudit.org/fr/usagers/politique-dutilisation/ 


\section{Paroisses françaises principales de la péninsule de Niagara}

Avant que de venir embrasser la frontière américaine, au milieu agité de la rivière Niagara, la province ontarienne est fortement coïncée, presque étranglée entre deux bouts de grands lacs : Erié et Ontario. Cet étranglement forme la péninsule de Niagara. En coupant la péninsule, dans sa partie extrême, du nord au sud, le merveilleux canal Welland crée une île artificielle, mais véritable puisque baignée tout autour par les grands lacs : Ontario au nord, Erié au sud, et la rivière Niagara à l'est et le canal Welland à l'ouest. Dans cette île se trouvent, éparpillées, une poignée de villes plus ou moins importantes : St. Catharines qui mène insensiblement à Merritton et Thorold, puis Niagara canadien en face de Niagara américain, Welland, Port Colborne et, face à Buffalo, Fort Erié.

Welland, c'est le nom d'une rivière qui traverse la péninsule de l'ouest à l'est et vient grossir le débit des chutes Niagara; Welland, c'est un canal merveilleux de 25 à 30 milles de long qui permet aux bateaux de passer d'un lac à l'autre par un escalier titanesque de huit marches liquides d'une hauteur moyenne de quarante pieds environ : les fameuses écluses de Thorold, véritable chef-d'œuvre du génie humain; Welland, enfin, c'est la ville traversée par la rivière et le canal du même nom, la ville où bat le cœur français de la péninsule.

Grâce au canal, aux chemins de fer : N.Y.C., C.N.R., C.P.R., sans parler des autoroutes voisines, les facilités de communications, à Welland, sont à peu près illimitées. Rien d'étonnant si de grandes et nombreuses usines y sont attirées. Vous y trouvez l' "Atlas Steel ", une des grandes aciéries du monde, dont les bâtisses noircies s'étalent sur une longueur d'un mille et demi; ajoutez deux grandes filatures de coton, une grande fabrique de tuyaux, "Le Page-Hersey », où fut coulé tout le pipeline de l'Alberta... bref une vingtaine de fonderies ou usines de toutes sortes, font peut-être de Welland, eu égard à son étendue et sa population, la ville la plus industrielle du Canada.

Cela expliquerait comment nombre de Canadiens français y soient venus d'un peu partout, attirés comme tout le monde par l'appât du travail varié et des bons salaires. Il en est venu de l'ouest, du nord, du sud même et surtout de l'est : Montmorency, Bellechasse et Gaspésie.

Avant 1915, on trouve bien peu de Canadiens français dans la péninsule. Tous ou à peu près y ont perdu leur langue, leur caractère ethnique, leur foi et jusqu'à leur nom. Nous avons encore un témoin du temps : M. Oliva Audet, ici en 1916. Vers 1917, l'immigration commence pour de bon pour atteindre bientôt le chiffre de quarante familles environ. Cette génération allait-elle aboutir au même résultat? On s'en effraya justement. 
La seule paroisse catholique, à cette époque, était St. Andrew's aujourd'hui St. Mary's. Le curé du temps était un apôtre zélé aux vues larges, vraiment catholiques, Fr. McCaffrey, irlandais. C'est lui d'abord, puis l'abbé Lussier, alors curé de Sainte-Jeanne-d'Arc de Toronto, et puis S.E. $\mathbf{M}^{\mathrm{gr}}$ O'Neil, archevêque de Toronto, qui virent la nécessité d'un prêtre de langue française. On s'en ouvrit à l'évêque de Saint-Hyacinthe, $M^{\mathrm{gr}}$ A.-X. Bernard, un saint homme, qui appela M. l'abbé R.-C. Tanguay. "Il y a, lui dit-il, un groupe canadien-français à Welland, Ontario, qui a besoin d'un prêtre bien à lui; je ne sais

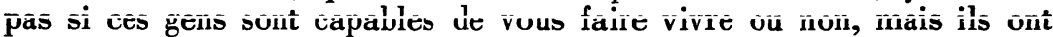
besoin d'un des leurs pour les aider dans leur situation. Je ne puis pas vous obliger d'y aller, mais je sais qu'il y a là une belle æuvre à faire. Est-ce que je puis compter sur vous? M. l'abbé Tanguay répondit à l'appel et la paroisse canadienne-française du Sacré-Cœur fut fondée.

Cette paroisse commençait à zéro, en octobre 1919, dans le soussol de l'église anglaise St. Mary's. En 1920, on achète un terrain sur la rue Queen, aujourd'hui Empire. Sur ce terrain, il y avait deux maisons dont la plus petite servit de presbytère et l'autre, d'église temporaire. L'église, ou plutôt la chapelle, fut vite jugée trop petite. On lui accola une vieille baraque militaire qui servirait en même temps de salle paroissiale. On appela cette salle " le camp ". Là on s'amusait, on dansait, on se rencontrait et on y entendait la messe, au moins à l'abri des intempéries sinon des rats et souris.

En 1934, on parle de bâtir une église. Monseigneur McNeil dit au curé : "Go ahead, father. Votre peuple en a besoin. Vos Français sont des bâtisseurs d'églises et ils paient leur église. Marchez, bâtissez ... » et l'on bâtit, à force d'énergie tenace et généreuse, de dévouement et d'économie, un temple qui comptait parmi les belles églises de la région.

Le 28 octobre 1934, S.E. $M^{\text {gr }}$ Decelles, évêque de Saint-Hyacinthe. sur l'invitation gracieuse de $\mathrm{M}^{\mathrm{gr}} \mathrm{Ch}$. Carroll, alors administrateur du diocèse de Toronto, procède à la bénédiction solennelle de la nouvelle église remplie à pleine capacité de Canadiens français auxquels se joignaient les catholiques marquants et les notables de la ville de toutes races et de toutes langues.

Malheureusement, avec la dépression tenace, ce fut la dispersion du troupeau qui baissa jusqu'à vingt-sept familles. C'était fort inquiétant pour le curé qui connut alors de bien dures épreuves. Dans le marasme du chômage, la pauvreté, la misère, l'impossibilité de payer même les intérêts, les dissensions apparurent ... Après avoir accompli une œuvre d'apôtre géant, l'inoubliable curé R.-C. Tanguay, dont la marque puissante reste encore visible, mourut d'une syncope, sur les bords du lac Erié, en juillet 1939.

Le nouvel archevêque de Toronto, S.E. $\mathrm{M}^{\mathrm{gr}}$ J. C. McGuigan supplia les Franciscains de se charger de la paroisse affligée, endeuillée, plus pauvre que jamais et pesant lourd sur ses épaules et son cœur... et c'est ainsi que les fils de saint François parurent dans la péninsule. 
- A quelque chose malheur est bon. " La deuxième grande guerre était commencée. Il fallait pousser la production jusqu'au bout. Le travail reprit, la main-d'œuvre manquait et la prospérité reparut. Avec elle reviennent peu à peu les familles dispersées dont le bonheur en attira d'autres de plus en plus : la paroisse reprend sa marche en avant.

En attendant l'arrivée des Franciscains, M. l'abbé Beauregard, père des orphelins agricoles de St. Catharines, plus tard Georgetown, aujourd'hui décédé, se chargea de l'intérim à la tête de la paroisse. Enfin, précédé de quelques mois par le R.P. Ildefonse Riopel, o.f.m., le premier curé franciscain apparut. C'était le R.P. André Godmer, o.f.m., qui deviendra plus tard missionnaire au Pérou où il trouva la mort, noyé dans l'Amazone. Ici le père accomplit sa tâche durant deux ans, dans un milieu divisé et troublé. Il est remplacé, en 1942, par le R.P. Louis-Joseph Bouchard, o.f.m., qui baissa la dette considérablement, décora l'église et mourut au poste en janvier 1948, l'année du chapitre, après avoir dit à ses amis peu auparavant : "Si je savais être changé j'aimerais autant mourir. " Il laissa d'ailleurs un excellent souvenir à Welland. Jusqu'au chapitre de 1948, le R.P. Marc Brunelle, o.f.m., vicaire, fut chargé de l'administration. Il faudrait une longue liste pour énumérer tous les vicaires qui se sont succédé à Welland et dont plusieurs se trouvent au Pérou.

En août 1948, le chapitre vint me chercher à la cure splendide de Notre-Dame, Trois-Rivières, pour me nommer curé de Welland. Je quittais pour l'inconnu une paroisse vieille, considérable et parfaitement organisée. J'avais l'impression nette de quitter tout pour tomber à rien. Je connaissais mal Welland. A la reprise des activités en septembre, ma retraite terminée, embrasé d'un zèle nouveau, je me mis à l'œuvre et, bientôt, ce qui m'avait paru amer s'était changé en douceur pour l'âme et pour le corps. Je fus complètement pris et conquis par Welland à tel point que je ne saurais plus comment en sortir même si l'on m'offrait de rentrer dans la paroisse idéale que j'ai tant aimée : Notre-Dame de Trois-Rivières.

Laissons-là maintenant les pasteurs et regardons grandir le troupeau. Les quelque 30 à 40 familles que nous avions prises en 1941 sont devenues rapidement $300,400,700,1,000,1,200$ et aujourd'hui 1,400 pour qui il fallait une grande église, où nous sommes, et dont je n'ai pas à vous faire l'histoire.

Si nos Canadiens français de Welland sont sauvés, ils le doivent à leur église d'abord, à leur école ensuite. La question scolaire est vitale partout, en Ontario comme ailleurs, sinon plus qu'ailleurs. Pas d'école française au moins bilingue, pas de paroisse française viable. L'héroïque curé R.-C. Tanguay l'a compris dès le début. Il avait obtenu une autre baraque militaire munie par le coton de quatre allées de quilles qui firent durant quelques années les délices d'une ligue dont on trouve encore les livres, registres, etc., chez M. O. Audet.

Tout d'abord, dans la première baraque militaire, le camp comme on l'appelait, M. Tanguay fit donner des leçons de français et de cathéchisme par deux admirables demoiselles, feu Anne-Marie (plus tard 
Mme Henri Arsenault) et Mlle Durocher. Ces deux institutrices recevaient pour tout salaire ce que l'on quêtait pour elles de porte en porte. Devant pareil dévouement, chapeaux bas, messieurs, et comme vous n'avez pas de chapeaux, applaudissez !

Pareil régime ne pouvait tenir longtemps. A force de délégations, de démarches et de pourparlers, la Commission scolaire anglo-protestante finit par se charger de l'école. La Commission scolaire couvrit d'un plancher les allées de quilles et y installa une classe. L'école étant trop petite, la Commission scolaire acheta, tout près, une usine de bas désaffectée qui se transforma vite en école convenable, Coronation School, une école publique où le principal, les professeurs et tous les élèves sont catholiques et Canadiens français, où l'on enseigne le français et le catéchisme sous l'œil d'un inspecteur protestant et d'une commission protestante, comme dans une école des frères, chose rare sinon inouie en Ontario.

En ce temps-là et jusqu'en 1959 ou 1960, Welland était séparé de Crowland. La population canadienne-française de Crowland grossissait à vue d'œil et en vint à égaler, peut-être même à dépasser, celle de Welland. Crowland était jaloux, et avec raison, de la situation faite aux Canadiens français de Welland. Il fallait faire quelque chose. Déjà, à mon arrivée en 1948, mon prédécesseur, feu le R.P. Louis-Joseph Bouchard, o.f.m., avait mandé les Sœurs du Sacré-Coeur et leur avait confié l'école maternelle privée. Pour aider Crowland on voulut prolonger cette école jusqu'en $5^{\circ}$ et $6^{\circ}$ années et même au-delà. Pour cela. il fallait agrandir et voilà comment on transporta les deux baraques pour les transformer en dix beaux logements et on les remplaça par l'école actuelle du Sacré-Cour. Bientôt cette école allait enseigner jusqu'à la $10^{\circ}$ année. C'était évidemment une école privée où les enfants devaient payer $\$ 5.00$ par mois. Tout cela ne pouvait satisfaire qu'en partie Crowland où le français n'était plus enseigné passé la $4^{\circ}$ année. Crowland étant township, on pouvait encore légalement lancer une commission scolaire bilingue. Et pourquoi pas? La Saint-Jean-Baptiste s'employa de son mieux. On songea d'abord aux écoles séparées. Après mûre délibération, on se demande si le système de Welland ne serait pas possible et plus pratique. Essayons. Une délégation ayant en tête le docteur Dubois rencontre la Commission scolaire de Crowland qui veut bien nous répondre favorablement et nous donner, dans Crowland, le système de Welland. Et voilà notre école élémentaire bilingue complètement organisée. Il s'agissait maintenant d'avoir notre inspecteur bilingue. Et nous l'avons dès 1960, grâce au travail du docteur Dubois et grâce aussi à la fusion des deux municipalités : Crowland et Welland.

Il fallait maintenant organiser le secondaire bilingue. Première solution : obtenir du High School Notre-Dame les classes bilingues nécessaires. J'ai fait moi-même les démarches auprès des autorités ici et à Montréal. Pour faciliter la chose j'offre audacieusement de payer le salaire annuel d'un professeur. La chose étudiée nous a été complètement refusée. Deuxième solution: le High School public. Le docteur Dubois accepte de faire les démarches auprès de la commission scolaire. Réponse : si le Départemnt accepte la chose, nous acceptons. Or le 
Département a déjà édité les programmes de High School bilingues. Donc, on accepte. Une condition : assurer une classe de quarante élèves. Ce que nous avons fait deux années consécutives. La troisième année, à cause des entrées trop nombreuses à la section technique où il n'y a pas de français prévu, nos classes bilingues ont vivoté et disparu.

Troisième solution: école privée. L'école à la fois primaire et secondaire, l'école du Sacré-Cour, devint exclusivement secondaire et les premiers de $12^{\circ}$ sortent cette année pour la première fois. Pour 1964 on promet un High School au moins, pour les filles, avec les Sœurs du Sacré-Cœur, probablement pour les garçons aussi avec les Frères qui voudront bien venir.

Pour compléter l'histoire scolaire de Welland, vous dirai-je, au grand ébahissement scandalisé de ceux qui ne connaissent ni le système ni le milieu, que nous avons refusé de faire partie des écoles séparées de Welland récemment organisées. Dans la nouvelle commission scolaire divisée en six sections avec deux commissaires par section, sur douze commissaires, deux de langue française seulement étaient assurés. alors que le nombre de nos enfants canadiens-français égalait à peu près le nombre de tous les autres enfants catholiques réunis. Les notables de paroisse consultés trouvèrent, peut-être avec raison, que notre système actuel offrait plus de sécurité et nous le gardons à moins que les autorités ne nous obligent à marcher avec les autres.

\section{PORT COLBORNE}

En l'an 1950, à Welland, je rencontrais pour la première fois Daniel Léveillé, citoyen de Port Colborne, qui me demandait s'il n'y aurait pas possibilité d'avoir un service religieux (messe, sermon, confession), au moins mensuel pour les Canadiens français de Port Colborne.

- Sont-ils nombreux ?

- Pas mal.

- Combien à peu près?

- Environ deux cents familles.

- En ce cas, faites signer une requête et je me charge de la présenter au cardinal McGuigan.

Ce ne fut pas long. Bientôt on me remit une requête qui avait recueilli environ deux cent cinquante signatures. Je me rendis auprès du cardinal qui regarda la requête, surpris de voir les Canadiens français aussi nombreux à Port.

- Etes-vous sûr du bien-fondé de cette requête?

- Je le crois, Eminence.

- En ce cas, ce n'est pas un service mensuel qu'il faut, ni même un vicaire canadien-français qui se plaindra toujours de son curé parce que celui-ci ne lui laissera pas assez de latitude. Ce qu'il faut, c'est une 
paroisse. Mais voilà ! Je n'ai personne à mettre là. A moins que les Franciscains n'acceptent de s'en charger. Qu'en pensez-vous ?

- Moi, Eminence, je suis prêt à favoriser la chose. Mais je ne puis rien décider. Il faut voir mon Provincial. Sans doute qu'une belle lettre de Votre Eminence aiderait beaucoup.

Il appelle immédiatement sa secrétaire et lui dicte une belle lettre que Son Eminence me charge de remettre à mon T.R.P.

Après avoir lu la lettre, le T.R.P. me dit à son tour, comme toujours :

- Je n'ai personne à mettre là.

Peu de temps après, je rencontre à Welland le R.P. Adélard Berthold, o.f.m., qui s'arrêtait me voir, obligé de quitter l'Ouest canadien et de rentrer en Corse, un peu déconcerté. Voilà mon homme, me dis-je.

- Dites, père Adélard, accepteriez-vous de fonder une paroisse canadienne-française tout près d'ici, à Port ?

- Oh oui ! et avec joie.

- C'est fait. Je donne votre nom au T.R.P. Provincial.

Le T.R.P. accepte et nomme le père Adélard, curé de Port Colborne.

Toute accommodation avec les paroisses anglaises s'est avérée impossible. Il fallut louer une salle. D'abord, deux ou trois dimanches, celle de la Légion qui nous réclamait $\$ 25.00$ pour utiliser, le dimanche matin seulement, son local laissé sale comme les écuries d'Augias. Il fallait nettoyer et préparer la salle pour les messes et quitter à midi.

On utilisa de même la salle hongroise de la rue Durham, à meilleur marché, mais trop petite. Enfin tout près de là, on s'installa pour un an dans la salle dite de la «Co-op ». Nous devions payer là un loyer assez élevé mais du moins étions-nous chez nous. Que de dévouement chez nos paroissiens pour préparer cette salle sans d'autre entrepôt que la cave pour les bancs, les tables, etc., avec des organisations fort disparates : messes, bingos, cartes, séances, réunions, etc.

Enfin on acheta le beau terrain actuel d'une famille italienne, Angelo Peressotti, au prix de $\$ 25,000$. Une grosse maison bâtie sur le terrain servit et sert encore de presbytère fort confortable. Tout à côté, on se mit en frais de construire au moins le sous-sol de l'église, d'après les plans à peu près gratuits d'un vieil architecte montréalais, $M$. Lemieux. La pierre angulaire est marquée de l'an 1952.

Le sous-sol à peu près terminé, le 3 mars 1953, on procéda à la bénédiction présidée par Son Eminence le cardinal McGuigan, assisté par le T.R.P. Hervé Blais, provincial, o.f.m. Je donnais le mot de circonstance, en l'absence du curé, le R.P. Adélard Berthold, o.f.m.

Ce jour-là, c'était le dimanche, on y disait la messe pour la première fois. L'honneur était réservé au R.P. Adélard dont le dévouement 
hérö̈que avait tout mené à bonne fin. On eût dit que le bon Dieu le gardait pour cela. En effet, le père Adélard célébrait la première messe dans l'église, mais, par contre, ce fut la dernière de sa vie. Durant la messe, le mal dont il souffrait déjà s'aggrava et, après la messe, il devint impotent et le resta jusqu'en juillet de la même année où il mourut à Montréal.

Pour lui succéder, le T.R.P. fit appel au R.P. Dieudonné Massé, o.f.m. qui marqua profondément son passage ici. La lutte scolaire allait commencer pour durer au moins trois ans, presque une autre grande guerre aux péripéties nombreuses, variées et tristes.

Déjà avant sa mort, le père Adélard avait demandé à la commission scolaire séparée des classes bilingues. Refus catégorique. Il fallait s'y attendre, mais ne pas lâcher. Avant l'arrivée du père Dieudonné, j'ai dû m'en mêler et mettre la commission scolaire au pied du mur. "Votre mandat, lui dis-je, est de donner l'école catholique aux enfants qui la réclament. Nous avons présentement environ deux cents enfants qui réclament et qui sont dans les écoles publiques. Vous n'avez pas de local. Nous vous offrons gratuitement notre sous-sol au moins pour lancer notre école bilingue. Vous manquez de finance. Durant au moins un an, pour vous aider, nous vous paierons la différence entre le coût d'opération et le revenu des octrois. Devant pareille offre, l'opposition croula et l'école bilingue s'ouvrit en septembre 1953 dans notre sous-sol, devenu tour à tour église, salle paroissiale et école avec six classes.

Evidemment, il fallait bâtir. La commission scolaire lésina à bâtir, pour six classes d'abord, notre école Saint-Joseph dont le nom remplaçait tout d'un coup sans avis celui de Berthold. Devant le flot d'élèves qui grossit jusqu'à plus de quatre cents, il fallait agrandir. On se laissera tirer l'oreille. Nos "pea-soup " nous laissent leur sous-sol gratuit, ils paient la différence entre le coût et l'octroi. Pour nous soulager, ils ont ouvert leur école rurale, l'école Massé; attendons.

Il fallut donc faire un coup d'état. Le R.P. Dieudonné Massé était de taille à l'organiser et il le fit. A l'ouverture des classes en septembre 1957, à Port Colborne, grand scandale aux yeux des Irlandais : le sous-sol est fermé. A moins d'entente avec la commission scolaire, les enfants sont dehors et n'ont plus d'école. Les enfants manifestent avec les parents accourus en nombre considérable. On se promène autour du sous-sol en criant, chantant et récitant le chapelet : "Nous voulons notre école bilingue."

L'inspecteur Kuska est averti. Il réunit au presbytère la commission scolaire. On discute chaudement, on en vient aux gros mots. L'inspecteur donne son avis : "A n'importe quelle condition, il faut faire quelque chose. „ Le père Dieudonné donne ses conditions : le sous-sol ne s'ouvrira que sous la promesse ferme, devant témoins, de payer les déboursés encourus par nous depuis le début, à savoir $\$ 15,000$. et de continuer ensuite de payer loyer moyennant $\$ 40.00$ par classe. On dut acquiescer et on acquiesça. Et la porte du sous-sol s'ouvrit toute grande. 
Malheureusement, entre-temps, le chapitre des Franciscains s'ouvre, les curés sont changés, moi-même je quitte Welland pour succéder au R.P. D. Massé. La commission scolaire refuse de faire honneur à sa parole et on dut accepter un règlement de comptes à $\$ 1,200$. à moins de renouveler le scandale. Je ne m'en sens pas la force et je n'ose le faire. Par contre, depuis, nos relations avec la commission scolaire s'adoucissent, deviennent même cordiales et tout va bien.

Grâce au concours de nos bonnes Sœurs du Sacré-Cœur, on ouvrit un pensionnat, on fonda même l'acadénie Saini-Lưuìis d’’ừ sônt sôrtics déjà trois classes de gradués mixtes en $12^{\circ}$ année.

D'ici deux ans, Port Colborne peut entrevoir la construction de son église. Je suis à étudier les plans, peu encouragé par ce qui šest passé pour l'église de Welland.

\section{NIAGARA}

Au moment de la bénédiction du sous-sol, à Port Colborne, Son Eminence le cardinal McGuigan s'enquit de la situation canadienne-française à Niagara. "Eminence, lui dis-je, la population canadienne-française à Niagara est au moins le double de celle de Port Colborne. C'est vrai que les travaux de l'Hydro-Ontario ont attiré beaucoup de Canadiens français qui disparaîtront, les travaux terminés, pour laisser à Niagara un noyau stable à peu près équivalent à celui de Port Colborne. Mais ne serait-il pas sage de profiter du nombre actuel de la population pour lancer une paroisse qui s'imposera un jour ou l'autre?...» * Alors, repartit Son Eminence, il faut une paroisse, mais je n'ai personne à y mettre à moins que les Franciscains n'acceptent de s'en charger. " "Eminence, le T.R.P. Provincial est ici et c'est entre vous deux seulement que la chose peut se décider. " En principe la paroisse est acceptée et décidée. Mais hélas ! les travaux étaient déjà terminés et les Canadiens français repartis lorsque le T.R.P. Théodoric Paré, succédant au T.R.P. Hervé, nomma curé de Niagara le R.P. Hilaire Tardif, o.f.m.

Le R.P. Hilaire accomplit en fort peu de temps un travail de géant. Un brave paroissien de Niagara, Hilaire Brideau, aménagea sa grande maison, sa cave et mit le tout fort généreusement, bénévolement et gracieusement à la disposition de la paroisse naissante. Il logea à la fois le bon Dieu et le curé, pendant au moins un an. Durant ce temps. en plein centre de la ville, on dénicha un fort beau terrain de 15 acres chargé de pêches et de fruits de toutes sortes et à fort bon compte. On acquit le terrain où bientôt s'éleva la belle église Saint-Antoine, pouvant accommoder sept cents personnes, munie d'un orgue Casavant fort enviable.

Entre-temps, le père Hilaire s'occupa sérieusement de la question scolaire. C'est dire qu'on a dû, comme toujours, lutter. Cette lutte, racontée dans ses détails absolument authentiques par un reporter du Globe and Mail, a fait scandale au point d'amener l'intervention austère de Son Eminence. Ce scandale apparent dont nous n'étions absolument pas responsables, joint au supposé scandale précédent de Port Colborne, 
tout cela fit tomber les clôtures et les barrières et l'école bilingue de Niagara fut fondée.

Le R.P. Hilaire s'épuisa vite à la tâche. Malade, il dut donner sa démission. Le R.P. Honoré Guilbert, o.f.m., prit la succession avec une ardeur et un dévouement admirables. Il acheta la propriété voisine dont il fit de la maison son presbytère, élargissant ainsi l'entrée du terrain trop étroite sur la rue, Portage Road. En peu de temps s'élevèrent l'école et la maison des sœurs. L'organisation est déjà à peu près complète, mais la dette de $\$ 255,000$. pèse bien lourd sur les épaules allègres d'une excellente population.

\section{HAMILTON}

Durant mon séjour à Welland, une délégation d'Hamilton, comprenant MM. Rozon, Marcotte et Plouffe, me demandait quoi faire pour lancer une paroisse canadienne-française à Hamilton où le chiffre de la population de langue française était estimé à environ dix mille. J'acceptai de m'en occuper. Je vis d'abord l'évêque avec qui la question fut discutée. Sur son avis, on fit circuler une pétition parmi les chefs de famille. Le nombre des signatures s'éleva à plus de trois cents. J'en donnai les résultats à l'évêque et l'on attendit. Un silence long et inquiétant s'ensuivit. Soudain le Saint-Esprit souffla et les grands journaux publièrent la grande nouvelle tant attendue : S.E. $M^{\mathrm{gr}}$ Ryan avait décidé la fondation d'une paroisse canadienne-française sous le vocable de Saint-Charles-Garnier.

Il faut admettre que la main de Son Excellence fut des plus heureuses dans le choix du premier curé. Father Murphy fit sur ses paroissiens une impression profonde, sympathique et qui dure encore. Ces dernières années, il y a trois ans au moins, Son Excellence Monseigneur l'Evêque mit le comble aux vœux des paroissiens en invitant les fameux pères de Sainte-Anne, les Rédemptoristes, à succéder au père Murphy promu justement à la belle cure de Brantford. Et je laisse au R.P. Simard le soin de continuer mon histoire.

Augustin Buisson, o.f.m.

Welland, Ont. 